\title{
Optimized FIB Sample Preparation for Atomic Resolution Analytical STEM at Low kV - a Key Requirement for Successful Application
}

\author{
M. Schaffer, ${ }^{* * * *}$ B. Schaffer, ${ }^{* * * * *}$ Q. Ramasse, ${ }^{* * * *}$ M. Falke, ${ }^{* * * *}$ D. Abou-Ras, ${ }^{* * * * *}$ \\ S.S. Schmidt, ${ }^{* * * * *}$ R. Caballero, ${ }^{* * * * *}$ and K. Marquardt, $* * * * * *$ \\ * SuperSTEM, STFC Daresbury Laboratories, Keckwick Lane, Warrington, WA4 4AD, UK \\ ** Department of Engineering, George Holt Building, Ashton Street, Liverpool, L69 3BX, UK \\ *** Kelvin Nanocharacterisation Centre, SUPA School of Physics and Astronomy, University of \\ Glasgow, Glasgow, G12 8QQ, Scotland, UK \\ **** Bruker Nano GmbH, Schwarzschildstrasse 12, 12489 Berlin, Germany \\ ***** Helmholtz-Zentrum Berlin, Hahn-Meitner-Platz 1, 14109 Berlin, Germany \\ ****** GFZ German Research Centre for Geosciences, Telegrafenberg, 14473 Potsdam, Germany
}

The focused ion beam (FIB) instrument is well established as a powerful sample preparation tool for materials analysis. The unique ability of FIB instruments to prepare smallest sample volumes from exactly defined areas of interest make them perfect and invaluable for preparation of thin lamellas in both, cross-section and plan-view configurations, for transmission electron microscopy (TEM) and scanning transmission electron microscopy (STEM) [1,2]. Within the last decade, several different FIB sample preparation techniques were developed and steadily improved, and their advantages and differences have been reviewed [3].

During the same period, TEM techniques have made a great leap forward by the introduction of Cs correctors [4,5]. Sub angstrom image resolution and atomically resolved electron energy-loss spectroscopy (EELS) allow new questions of materials science to be tackled. However, such outstanding results can only be obtained from samples of supreme quality regarding thickness, cleanliness and structural integrity. The recent development in different EM techniques towards lower acceleration voltages results in the need for even better samples. FIB preparation without subsequent sample treatment has often been hastily regarded as insufficient technique for atomic resolution STEM at low voltages. Typically, such investigations require a FIB prepared sample to achieve less than $30 \mathrm{~nm}$ thickness with a minimum of ion beam damage and material redeposition of sputtered material at the sidewalls.

In this contribution we will show that latest generation DualBeam FIB instruments with optimized preparation protocols and the capability of low-kV milling down to $0.5 \mathrm{kV}$ can meet these high expectations and provide samples for atomic resolution Cs-corrected STEM EELS analysis even at low voltages down to $60 \mathrm{kV}$. Specimen thickness between 15 and $30 \mathrm{~nm}$ can be achieved for a large variety of materials without loosing the unique advantages of FIB site specific and small-volume preparation. In addition, FIB preparation offers the advantage of minimizing sources of sample contamination, as glues are completely avoided. Additionally, specimen size is reduced in comparison to other preparation techniques, reducing the surface area from which contaminants may migrate towards the electron transparent section.

We will present results of interfaces in metal-disilicide/silicon systems (Fig. 1), grain boundary investigations in $\mathrm{Cu}(\mathrm{In}, \mathrm{Ga}) \mathrm{Se}_{2}$ based solar cell devices (Fig. 2), and target preparation for interdiffusion investigations in a YAG bi-crystal. The improved FIB preparation enabled high sensitivity work which was formerly not accessible with standard conventional and FIB sample preparation of the materials. All STEM investigations were performed with the NION UltraSTEM 100 at SuperSTEM/Daresbury UK. 


\section{References}

[1] L.A. Giannuzzi et al., Micron 30 (1999) 197.

[2] R.M. Langford et al., J. Vac. Sci. Technol. A 19 (2001) 2186.

[3] R.M. Langford et al., Microscopy Research and Technique 69 (2006) 538.

[4] M. Haider et al., Nature 392 (1998) 768.

[5] P.E. Batson et al., Nature 418 (2002) 617.

[6] The authors would like thank EPSRC for funding under grant EP/D040205/1. Durham University, University of Glasgow, University of Leeds and Technical University of Denmark are acknowledged for granting FIB access.
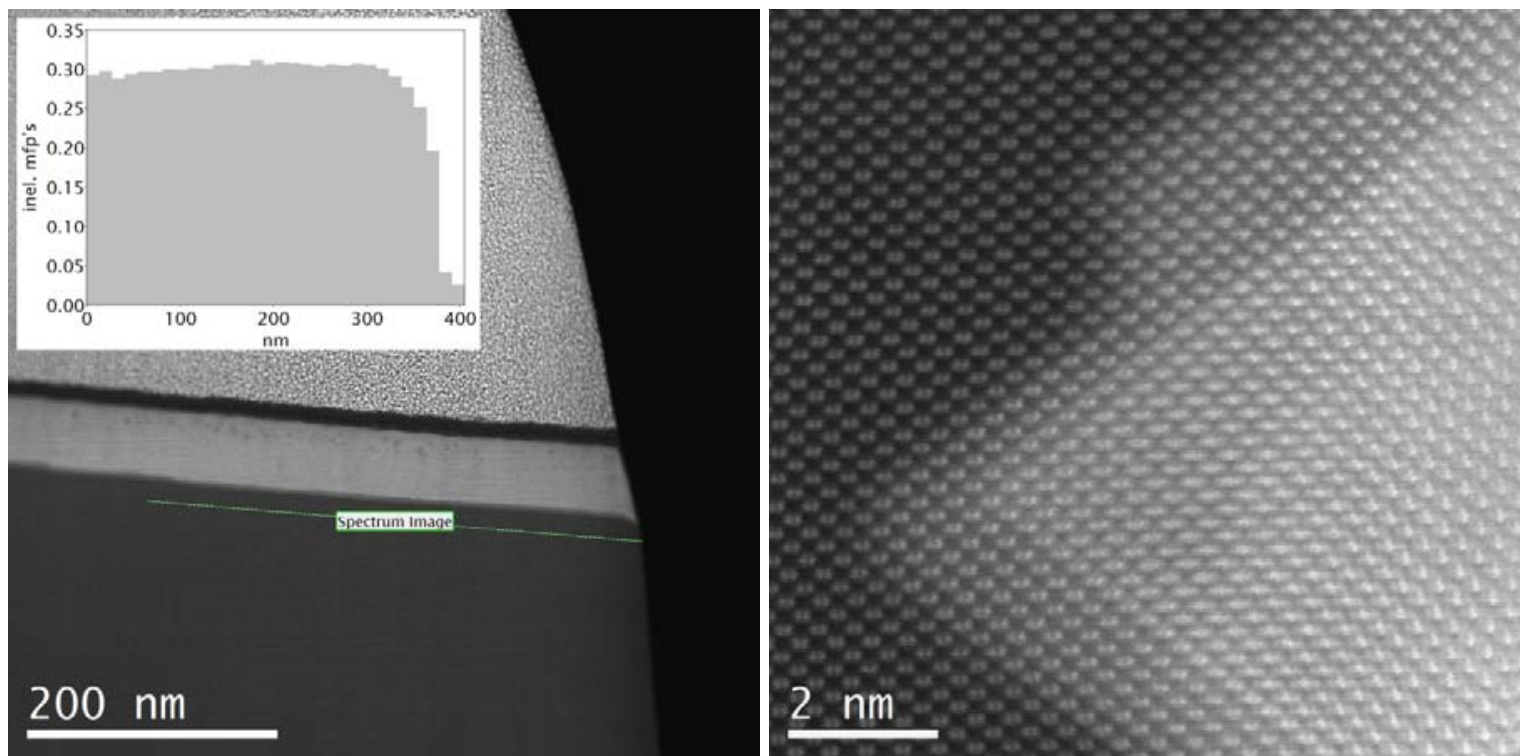

Fig. 1. HAADF images of a NiSi 2 / Si interface acquired from a FIB lamella of $\sim 22 \mathrm{~nm}$ thickness.
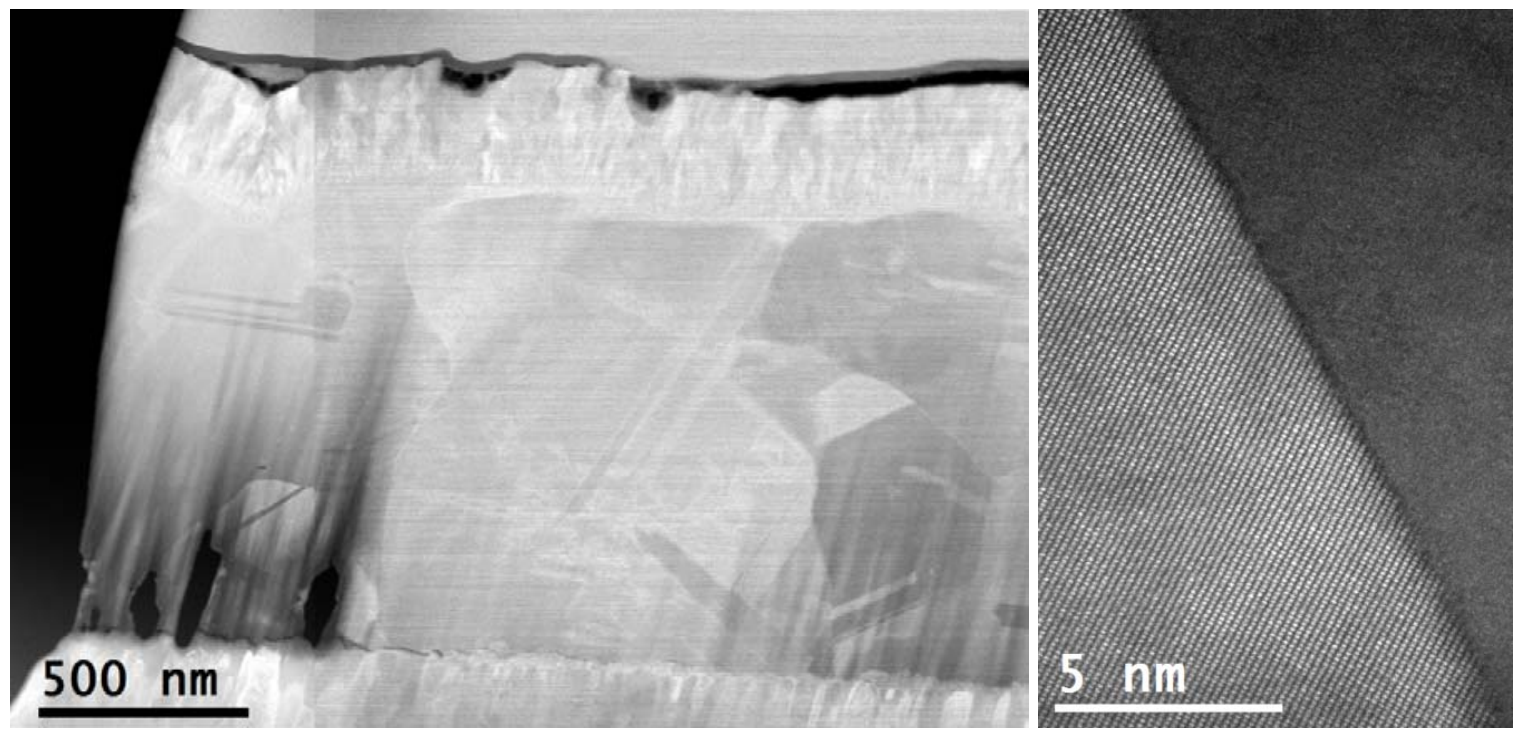

Fig. 2. MAADF overview and HAADF detail image of a non-twin grain boundary in the $\mathrm{Cu}(\mathrm{In}, \mathrm{Ga}) \mathrm{Se}_{2}$ absorber of a solar cell. The sample was prepared from a working device and areas of interest are thinned to $<15 \mathrm{~nm}$ thickness with sufficient undamaged crystalline material to give high quality HAADF images in Cs corrected STEM. 